\title{
Phase Separation and Dewetting Induced Surface Pattern in PS/PMMA Blend Films
}

\author{
Xiong RuI, ${ }^{\dagger}$ Zhiwei Song, Shi JING, and Tian Decheng \\ Department of Physics, Wuhan University, Wuhan 430072, China
}

(Received December 21, 2004; Accepted April 26, 2005; Published August 15, 2005)

\begin{abstract}
The pattern evolution of phase separation and dewetting of PS/PMMA blend thin films at $160{ }^{\circ} \mathrm{C}$ were observed by optical video microscopy and atomic force microscopy (AFM). The results show that the PMMA wetting component is driven toward the substrate, and the PS dewetting component is driven toward the surface by pressure caused by phase separation and wetting. At the late stage of the process, irregular shapes of PS islands evolve into round droplets. A cross-section of the interface between the PS and PMMA after phase separation was observed by AFM for the first time. This shows that the PS component extends considerably into the PMMA substrate layer and a trench was observed inside and outside the PMMA rim, respectively. This confirms the notion of a deformable interface between the two materials. [DOI 10.1295/polymj.37.560]

KEY WORDS PS/PMMA Blend Films / Phase Separation / Dewetting /
\end{abstract}

The structure and properties of thin blend films are governed by interplay between phase separation and surface segregation driven by polymer-surface interactions. This is important for applications because commercial films are normally comprised of multiple polymer species, solvent, and additives, leading to numerous complex phase separation morphologies and film properties. As a function of blend composition and polymer-polymer interaction parameter different phase separation morphologies have been observed in bulk samples. In the state of a thin film, with a thickness below a critical film thickness, the surface directed spinodal decomposition of polymers is suppressed and phase separation perpendicular to the interface results. The interplay between phase separation and dewetting increases the variety of accessible surface morphologies. The time scales of dewetting and phase separation are different and depend on the internal interaction. ${ }^{1-8}$ However, understanding of phase evolution in blend films has not yet been achieved in details, in part due to the complex interplay between wetting, phase separation, capillary fluctuations and coarsening and roughening, respectively.

Polystyrene (PS) and poly(methylmethacrylate) (PMMA) are classic model systems in polymer science, and numerous studies have been recently been undertaken..$^{9-12}$ This report studies the phase separation and dewetting of PS and PMMA blend films with different thickness, using optical video microscopy in situ during annealing. For the first time, we report the interface characteristic of PS/PMMA in the final stage of phase separation.

\section{EXPERIMENTAL}

First, PS and PMMA were dissolved in toluene. The polymers had molecular weights $M_{\mathrm{w}}=60500 \mathrm{~g} / \mathrm{mol}$ (PS), and $M_{\mathrm{w}}=62500 \mathrm{~g} / \mathrm{mol}$ (PMMA) and polydispersities $M_{\mathrm{w}} / M_{\mathrm{n}}=1.07(\mathrm{PS})$ and $M_{\mathrm{w}} / M_{\mathrm{n}}=1.12$ (PMMA). The total polymer concentration was prepared first at 3\% and then part of the solution was diluted to $2 \%$ and $1 \%$. The PS mass fraction $\phi$ was fixed at 0.50 . Thin films of the polymer blends were formed by placing a droplet of the solution onto $1 \mathrm{~cm} \times 1 \mathrm{~cm} \mathrm{Si}(001)$ with the native oxide layer present $\left(\mathrm{Si}-\mathrm{SiO}_{x}\right)$ and spinning it at high speed. Film thickness could be controlled by solution concentration and spinning speed. Then the blend films on $\mathrm{Si}$ substrates were annealed under vacuum at temperature $T=90^{\circ} \mathrm{C}$ (lower than the glass transition temperature $T_{\mathrm{g}}$ of both PS and PMMA) for $12 \mathrm{~h}$. This annealing removes residual solvent molecules which act as plasticizers and allows relaxation of chains.

The thickness of blend films ranging from $15 \mathrm{~nm}$ to $140 \mathrm{~nm}$ was measured using ellipsometry. Phase separation experiments were performed in two different ways. In the first, samples with different thickness were annealed at $160^{\circ} \mathrm{C}$ for $24 \mathrm{~h}$. The temperature is well above the glass transition temperature $T_{\mathrm{g}}$ but lower than that of the occurrence of degradation of the polymer molecule of both PS and PMMA. The morphologies of samples at the final state were recorded using optical microscopy and atomic force microscope (AFM). Secondly, dewetting and phase separation were performed on a hot-stage at $160^{\circ} \mathrm{C}$. The morphologies of dewetting and phase separation

†To whom correspondence should be addressed (E-mail: xiongrui @whu.edu.cn). 


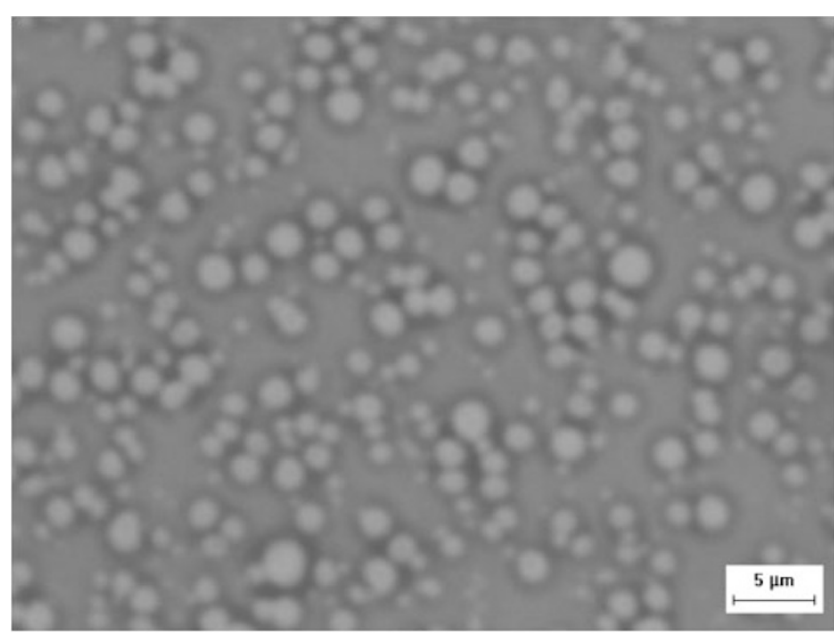

(a)

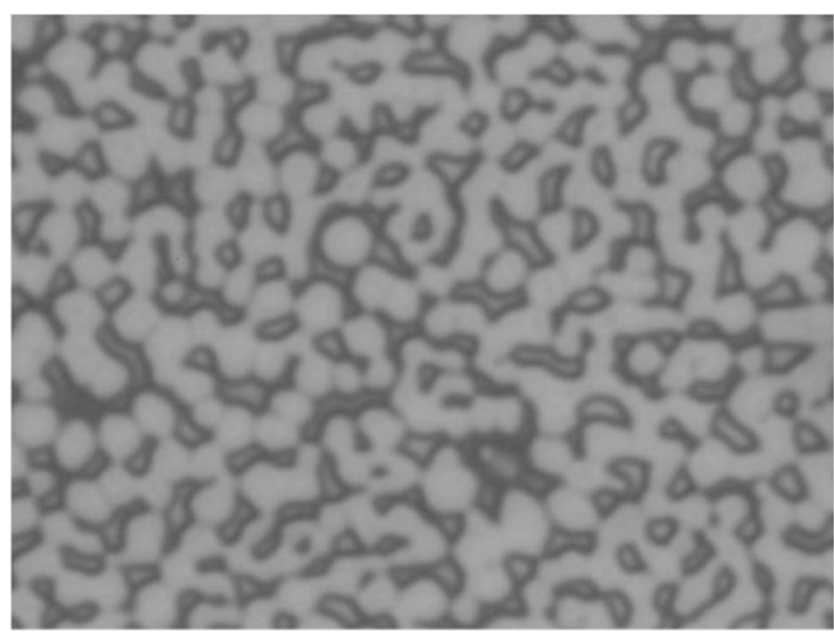

(b)

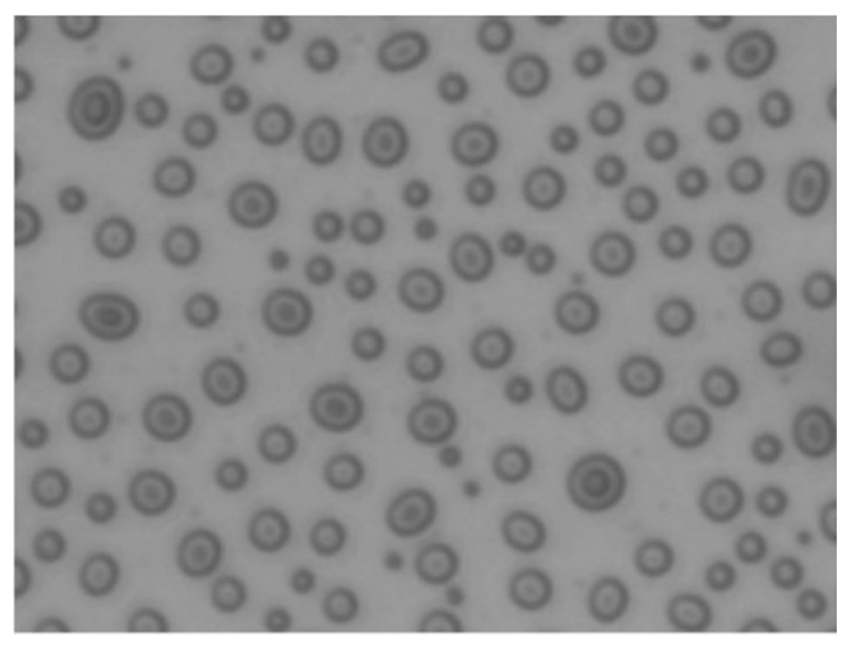

(c)

Figure 1. Pattern evolution of PS/PMMA blend films annealed at $160^{\circ} \mathrm{C}$ a) $30 \mathrm{~min}$, b) $180 \mathrm{~min} \mathrm{c)} 800 \mathrm{~min}$.

were observed by optical microscopy in the reflection mode, and each sample was annealed until morphology ceased to change.

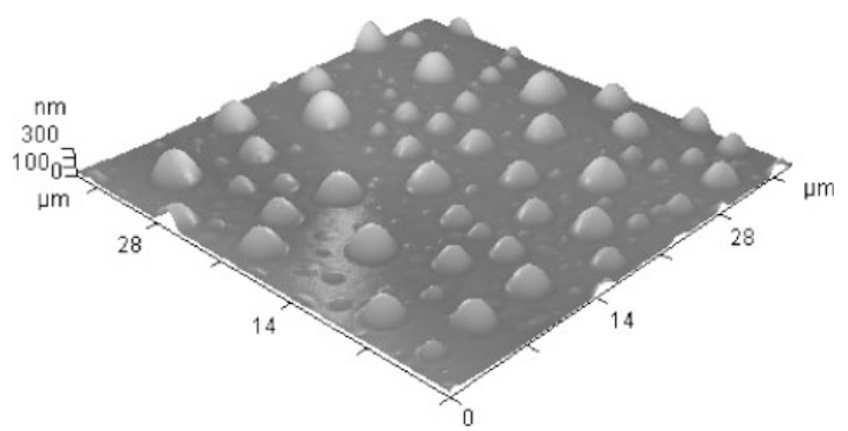

Figure 2. AFM image of the $50 \mathrm{~nm}$ PS/PMMA blend film.

Data analyses of the phase-separated films were done using an optical metallurgical microscope. The microscope was fitted with a CCD camera, which was interfaced through a frame grabber to a PC in order to obtain digital images. There was no need for staining the films to enhance the optical contrast of the components, since the difference in the index of refraction ( $\Delta \mathrm{n}=0.10$ for PS/PMMA) ensured that the two phases had different colour. Colour filters were used in some cases to increase the contrast. This method was sufficient for all films in the present study although the image quality was poor for very thin films $(15 \mathrm{~nm})$. The phases of the phase-separated films were identified unambiguously by selectively removing one of the polymer components. This was done by rinsing the film with a solvent for only one of the polymer components. All measurements were carried out at the center of the spin-coated films to minimize the effect of shear on the morphology.

\section{RESULTS AND DISCUSSION}

The time development optical micrographs of the surface pattern formation associated with the phase separation and dewetting could be divided into three stages. Figures 1a, b, and c show typical optical micrographs of these three stages. The film thickness before annealing was about $50 \mathrm{~nm}$. The scales of observation are indicated by the reference bar shown in Figure 1a. The phase separation and dewetting of other films with different thickness were morphologically similar. The phase separation pattern is formed at very early stage, but no obviously bicontinuous spinodal decomposition structure ${ }^{13-15}$ was observed in the early stage in these films. Colour change in Figure 1a is a result of phase separation and fluctuation in surface height.

Figure 2 is the AFM image of the $50 \mathrm{~nm}$ blend film annealed at $160^{\circ} \mathrm{C}$ for $24 \mathrm{~h}$. AFM shows isolated droplets $25 \mathrm{~nm}$ in height and $2-4 \mu \mathrm{m}$ in diameter arranged in an arraylike fashion. These droplets thus have a "pancake-like" form, and the aspect ratio, $\alpha$, 

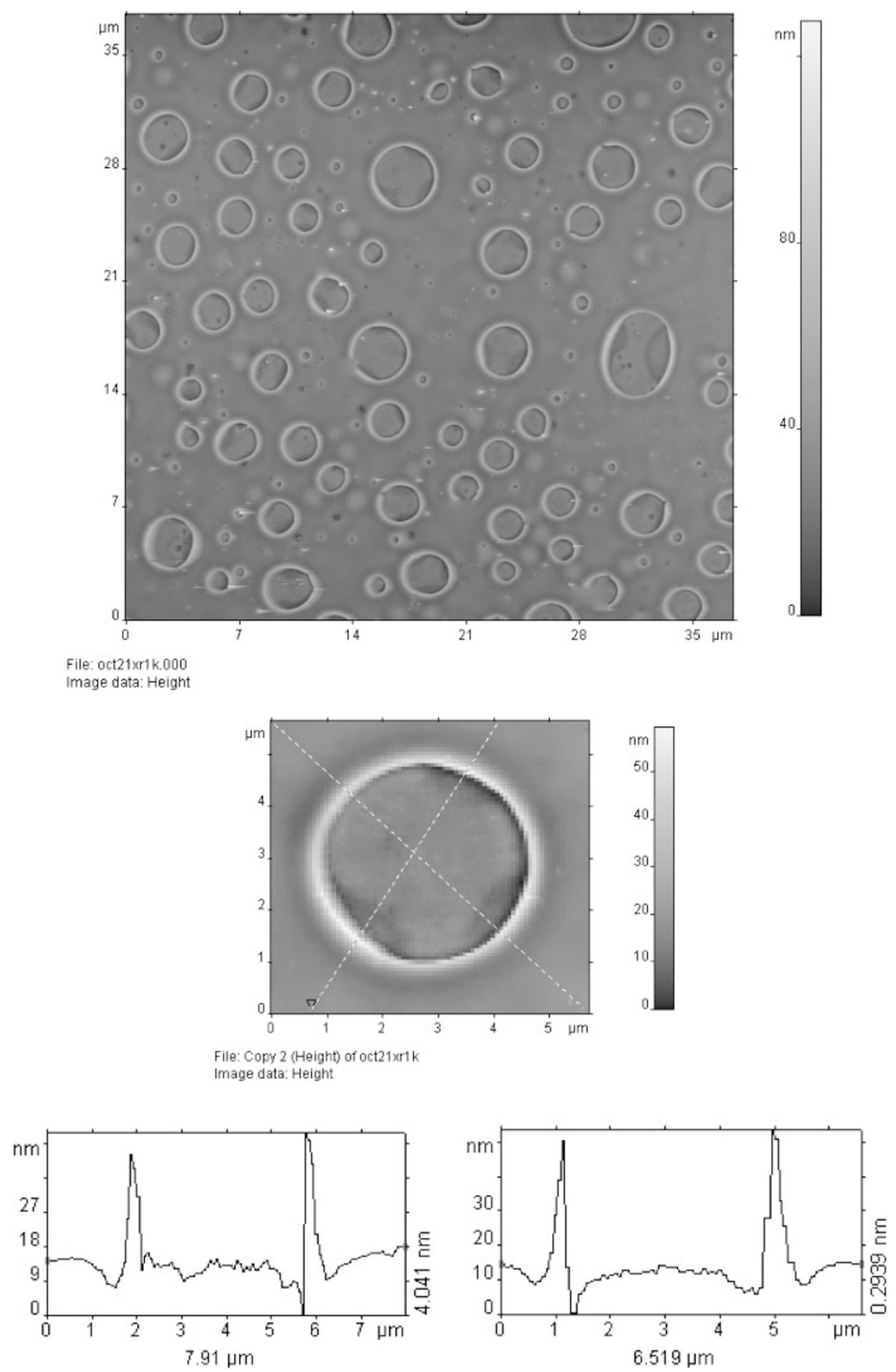

Figure 3. AFM image after PS component selectively removed by carbon tetrachloride.

the ratio of the height $h$ to the diameter $R$ of the droplets, is small $(\alpha=h / R \ll 1)$. This image is very similar to what was observed through optical microscopy in the very late stages of phase separation and dewetting.

To establish the shape of the PS/PMMA characteristic in the final surface pattern, PS was selectively removed by immersing the samples into carbon tetrachloride. After drying, the remaining PMMA surface was imaged with AFM again. The AFM is shown in
Figure 3. Comparing the two AFM images provides that all isolate droplets are made of PS and removed by immersing the samples into carbon tetrachloride. The cross-section of the AFM image at well defined location (generally through the center of the hole) after selective removal of the PS is also shown in Figure 3. The curved shape of the bottom part and the edge clearly shows that the PS component extends considerably into the PMMA substrate layer, thereby confirming the notion of a deformable interface 


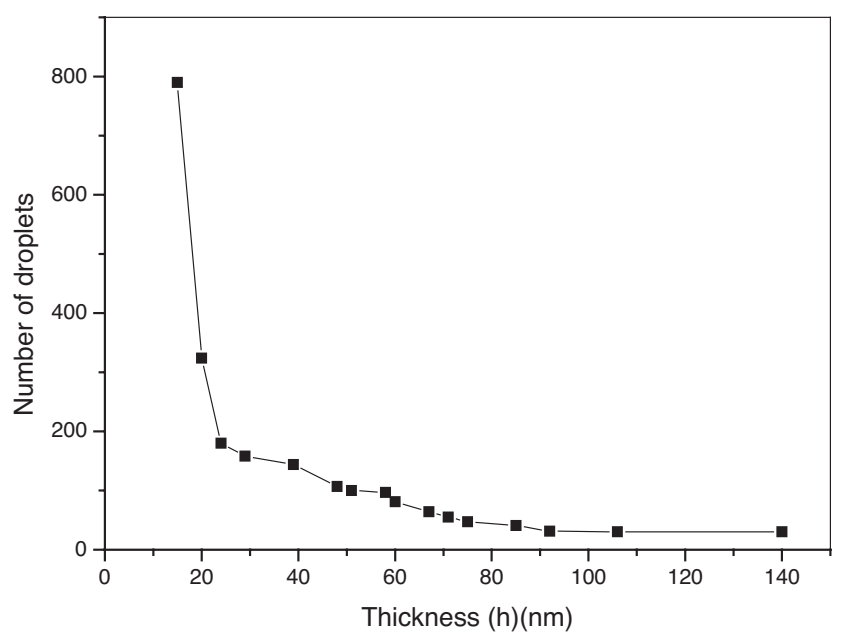

Figure 4. Dependences of the number of droplets per reference area on initial film thickness (h).

between the two materials. The PMMA rim shown in Figure 3 was much higher than the PMMA around the droplets. A trench was observed inside and outside the PMMA rim. The droplets of PS itself thus clearly extend into the PMMA layer below the PS/PMMA interface. The "pancake-like" droplets evidently have anisotropic structure. The interface characteristic is similar to that of PS dewetting on PMMA surface, ${ }^{11,12}$ although the final patterns have big differences possibly due to strong shear force from dewetting and phase separation. The AFM images indicate that the interface deformation is highly localized near the contact line.

By combining depth profiling and morphological data, the pattern evolution of phase separation and dewetting in PS/PMMA blend films can be described. Initially, the PS/PMMA blend phase forms a thin wetting layer at the surface and substrate. Clearly phase separation pattern forms at the very early stage when the film is annealed at $160{ }^{\circ} \mathrm{C}$. Different colours could be observed in Figure 1a. The white phase in Figure 1a may be the PMMA phase, connected to the wetting layer, and wets the substrates. Towards the end of the first stage, a very small PS phase (blue phase) extrudes from the film driving by the pressure of PMMA phase wetting the substrates. In the second stage, the PS phase becomes more and more bigger with time [Figure 1b]. In the end of this stage, the PMMA phase goes on the substrate, and irregular shape of PS islands flow on top of the PMMA layer. During the late stage, PS islands of irregular shape evolve into round droplets due to surface and interfacial energies of the two materials [Figure 1c].

Typical micrographs for the final patterns after phase separation and dewetting of blend films with different thickness demonstrate that the initial film thickness (h) influences significantly various parame-

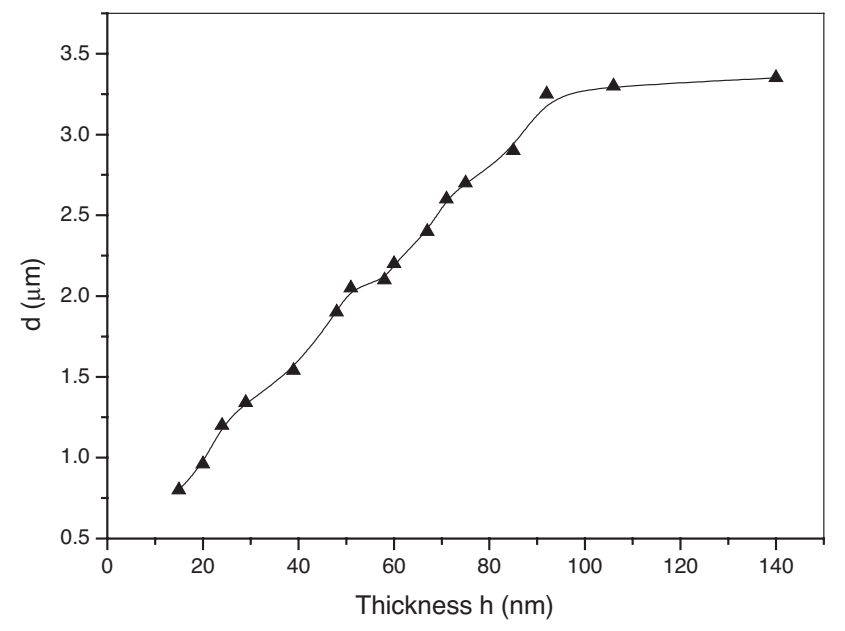

Figure 5. Dependences of typical size of final droplets per reference area on initial film thickness (h).

ters characterizing droplets and structures on the micrographs. The distribution of droplets is random and the size of droplets also is random. The dependences of the number and typical size of the final droplets per reference area on $\mathrm{h}$ are shown in Figure 4 and Figure 5. With increasing thickness of the blend films the droplets grow in size but decrease in number. As thickness increases close to $100 \mathrm{~nm}$, the number and typical size of the final droplets per reference area seem stable.

\section{CONCLUSIONS}

PS/PMMA blend films were prepared by spin coating. Phase separation and dewetting induced surface pattern were observed by optical video microscopy and atomic force microscopy. The pattern evolution of PS/PMMA blend thin films undergoing simultaneous phase separation and dewetting can be described. During the first and second stages, pressure due to phase separation and wetting drives the PMMA wetting component towards the substrate, and the PS dewetting component towards the surface. Towards the end of these two stages, PS islands of irregular shape flow on top of the PMMA layer. During the late stage, the flowing PS islands of irregular shape evolve into round droplets due to surface and interfacial energies of the two materials.

Cross-sections of the AFM image after selective removal of the PS component demonstrate that the PS component extends considerably into the PMMA substrate layer, confirming the notion of a deformable interface between the two materials. A trench was observed inside and outside the PMMA rim possibly due to strong shear force, which arises with the progress of dewetting and phase separation. 
Acknowledgment. We are indebted to the Scientific Research Foundation for the Returned Overseas Chinese Scholars, State Education Ministry. We thank Prof. John Dutcher and Dr. Oleg Stukolov of Guelph University of Canada for critical and valuable discussions over the work and assistance in the experiments.

\section{REFERENCES}

1. H. Wang, F. J. Douglas, S. K. Satija, J. R. Composto, and C. C. Han, Phys. Rev. E., 67, 61801 (2003).

2. M. Geoghegan, H. Ermer, G. Jungst, G. Krausch, and R. Brenn, Phys. Rev. E., 62, 940, (2000).

3. D. S Liu, L. X. Wang, S. J. Xie, S. H. Han, and L. M. Mei, Chin. Phys., 12(5), 548 (2003).

4. H. Tanaka, Phys. Rev. Lett., 70, 2770 (1993).

5. W. Feng, Y. L. Xu, W. H. Yi, F. Zhou, X. G. Wang, and K. Yoshino, Chin. Phys., 12(4), 426 (2003).

6. G. Krausch, C. A. Dai, E. J. Kramer, and F. S. Bates, Phys.
Rev. Lett., 71, 3669 (1993).

7. A. M. Higgins and R. A. L. Jones, Nature, 404, 476 (2000).

8. Z. Y. Liang, K. Fang, and X. Li, Chin. Phys., 9(11), 837 (2000).

9. A. M. Higgins, M. Msferrazza, R. A. L. Jones, P. C. Jukes, J. S. Sharp, L. E. Dryden, and J. Webster, Eur. Phys. J. E, 8, 137 (2002).

10. J. D. Gunton, M. San Miguel, and P. S. Sahni, "Phase Transitions and Critical Phenomena," C. Domb and J. L. Lebowitz Ed., Academic Press, London, U.K., 1983.

11. S. Qu, C. J. Clarke, Y. Liu, M. H. Rafailovich, J. Sokolov, K. C. Phelan, and G. Krausch, Macromolecules, 30, 3640 (1997).

12. F. Brochard-Wyart, P. Martin, and C. Redon, Langmuir, 9, 3682 (1993).

13. L. Sung, A. Karim, J. F. Douglas, and C. C. Han, Phys. Rev. Lett., 76, 4368 (1996).

14. H. Tanaka, J. Phys.: Condens. Matter, 13, 4637 (2001).

15. H. Wang and R. J. Composto, Macromolecules, 33, 3274 (2000). 